\title{
The Behavioral Effects of Frequent Nightmares on Objective Stress Tolerance
}

\author{
Kevin D. Hochard \\ University of Chester \\ Nadja Heym \\ Nottingham Trent University \\ Ellen Townsend \\ University of Nottingham
}

Frequent nightmares have been linked to daily distress using self-report measures. The present study investigated the impact of frequent nightmares on a stressful cognitive test requiring participants to perform additions of 2 previously displayed single digit numbers from a number series, where display latency between digits becomes increasingly short-the Paced Visual Serial Addition Task-Computerized $(P V S A T-C)$. Participants experiencing frequent nightmares $(n=43)$ and controls $(n=42)$ were compared on PVSAT-C performance. A significant main effect of nightmare frequency was observed with participants in the frequent nightmare group enduring the task for a shorter duration than controls (a behavioral measure of stress tolerance). Results suggest that individuals experiencing frequent nightmares have a reduced tolerance for stressors, leading to increased daily vulnerability to stressful stimuli. This study confirms previous findings linking nightmares and daily distress and extends the literature by providing objective evidence for the link between nightmares and reduced stress tolerance through behavioral testing. These findings highlight nightmares as a salient target for clinical intervention.

Keywords: resilience, stress, sleep, nightmares

Nielsen and Levin's (2007) affective network dysfunction (AND) model of nightmares is a clear account of nightmare formation rooted in neurophysiology, whereby nightmares are a dysfunction of naturally occurring fear memory extinction achieved through normal dreaming. More precisely, the current affective load experienced by an individual is said to dictate the need for the formation of new

Kevin D. Hochard, Department of Psychology, University of Chester; Nadja Heym, Division of Psychology, Nottingham Trent University; Ellen Townsend, School of Psychology, University of Nottingham.

Correspondence concerning this article should be addressed to Kevin D. Hochard, Department of Psychology, University of Chester, Parkgate Road, Chester, CH14BJ, United Kingdom. E-mail: k.hochard@chester.ac.uk 
fear extinction memories. Memories are thus created during the dream process by dissociating and recombining attributes of existing fear memories. These memories are then processed as a novel fear extinguishing stimuli. However, nightmares occur due to a failure within this process whereby the recombined memory is consistent to waking-state fear memories akin to phobias or social anxiety. Crucially, for the present study, the AND model suggests that nightmares reduce distress tolerance, namely, one's ability to resist stressful stimuli, due to a failure to regulate affect over a given sleep period leaving the individual more susceptible to stressors on awakening. In line with this model, frequent nightmares have been associated with increased daily distress and negative affect (Köthe \& Pietrowsky, 2001), reduced well-being (Blagrove, Farmer, \& Williams, 2004) and sleep distress, that is, tiredness upon waking, lack of energy during the day, irritability, worry about amount of sleep obtained, and perceptions of having slept poorly (Lancee \& Schrijnemaekers, 2013).

Existing literature exploring the impact of nightmares on behavior has thus far relied on self-reported measures. With the current trouble in psychology surrounding the lack of replicable findings (Open Science Collaboration, 2015), it is of critical importance that replication studies be performed and communicated to strengthen confidence in fundamental assumptions which guide the literature. With this in mind, the present study aims to measure the influence of frequent nightmares on tolerance to a cognitive stressor using a behavioral task, in order to replicate the findings of prior studies (Blagrove et al., 2004; Köthe \& Pietrowsky, 2001; Lancee \& Schrijnemaekers, 2013). The Paced Auditory Serial Addition Task (PASAT) - a mathematical summation task - has frequently been reported by participants to be highly stressful (Lejuez, Kahler, \& Brown, 2003). Its visual computerized variantPaced Visual Serial Addition Task-Computerized (PVSAT-C) - has been validated and used as a stress induction task to explore stress resilience in a variety of psychopathologies (Lejuez et al., 2003). Thus, an individual's threshold of stress tolerance can be measured behaviorally as the duration over which they are able to continue with the stressful task.

However, several factors could potentially influence PVSAT-C performance. Reviewing Paced (Auditory/Visual) Serial Addition Tasks, Tombaugh (2006) indicates mathematical ability as well as depression and high levels of anxiety as potential confounding variables. In addition, Hochard, Heym, and Townsend (2015) report increased negative affect and greater likelihood of engaging in self-harmful behaviors on the day following the occurrence of a nightmare, while Daughters et al. (2005) have shown that negative affect has a direct impact on PVSAT-C task performance. General poor sleep may also be an issue since sleep deprivation has been shown to have a deleterious effect on cortisol levels and stress tolerance (Leproult, Copinschi, Buxton, \& Van Cauter, 1997). It is therefore necessary to control for the occurrence of nightmares directly prior to engaging in the task since this short term state effect would likely mask our ability to detect the trait effect of frequent nightmares. Controlling for global sleep quality as well as mathematical ability, depressive symptoms, and anxiety would help alleviate concerns of confounding variables impacting on PVSAT-C performance.

Based on past literature (Blagrove et al., 2004; Köthe \& Pietrowsky, 2001; Lancee \& Schrijnemaekers, 2013), we hypothesize that participants experiencing 
frequent nightmares will be less tolerant to stress as measured by the PVSAT-C than participants experiencing infrequent or no nightmares.

\section{Method}

\section{Participants}

Eighty-five participants (12 males, 6 in each group) aged $18-31$ ( $M=20.95$ years, $S D=3.25$ ) were recruited from a pool of 382 university students who had previously completed a short online screening questionnaire regarding sleep issues. To be included in the study, participants could not be currently using psychoactive medication or have an existing psychiatric diagnosis. This study received ethical approval from the University of Nottingham School of Psychology Ethics Committee. Written consent was obtained at the screening stage and again prior to participation in the lab-based testing stage of the study. Research credits and a small monetary incentive were granted in exchange for participation. Participants were naïve to the hypothesis of this study. Debriefing information and resources to relevant health and mental health helplines were provided on completion of the study.

\section{Materials}

Participants were assessed for nightmares using the 7 item Disturbing Dream and Nightmare Severity Index (DDNSI; Krakow, 2006) which measures frequency of nights of nightmare per week (0-7), the number of nightmares experience per week (0-14), frequency of nightmare-related awakening $(0=$ never to $4=$ always $)$, the perceived severity of the problem $(0=$ no problem to $6=$ very severe $)$ and the experienced intensity of the nightmare $(0=$ not intense to $6=$ extremely severe intensity). Scores of $\geq 10$ on the DDNSI represent clinical levels of disturbing dreams and nightmares (Krakow, 2006) indicative of nightmare disorder (American Academy of Sleep Medicine, 2014). Using this cutoff, participants were categorized as belonging to the nightmare (nightmare disorder) or control (subclinical nightmares) group.

A nightmare recall questionnaire (binary variable: yes/no) assessed for the occurrence of a nightmare on the night prior to the study. This was used as a covariate to control for the potential confounding effect of a solitary nightmare on stress tolerance threshold.

The 21 item Beck Depression Inventory-II (BDI-II; Beck et al., 1996) measured the presence and severity of depressive symptoms over the previous 2 weeks. The BDI-II was used to control for the effects of depressive symptoms in the present study (Tombaugh, 2006).

The Pittsburgh Sleep Quality Index (PSQI; Buysse, Reynolds, Monk, Berman, \& Kupfer, 1989) assessed global sleep quality over the past months through seven components: sleep onset latency, subjective sleep quality, estimated sleep duration, sleep efficiency, sleep disturbances, use of sleep aids, and daytime dysfunction used 
as a covariate to control for the potential confounding effect of poor sleep quality on stress tolerance.

The revised Reinforcement Sensitivity Theory Behavioral Inhibition System Anxiety subscale (rRST BIS Anxiety; Heym, Skatova, Ferguson, \& Lawrence, 2015) assessed trait anxiety level which may alter performance on the PVSAT-C (Tombaugh, 2006).

The PVSAT-C (Lejuez et al., 2003) was used as a stress tolerance task. The task requires participants to perform serial additions using the two previously displayed single digits in a long running series of digits. The latency between digits begins at $3 \mathrm{~s}$ in the initial 3 min block allowing participants to acclimatize to the task itself. The second block is 5-min long with a shorter display latency of $2 \mathrm{~s}$ between digits. A 2-min mandatory rest period is given to participants prior to beginning final block. The third block has a latency of $1 \mathrm{~s}$ and can last for a maximum of 10 min; however, participants are instructed during the rest period that due to the difficulty they are allowed to end this block at any time by pressing a designated key on the terminals' keyboard. Duration of the third block provides a time measure for resistance to psychological stress. As the task is likely to be moderated by mathematical ability (Tombaugh, 2006), an objective measure of ability (highest math qualification: $0=$ none, $1=$ GCSE, $2=$ AS-level, $3=$ A-level, $4=1$ st degree) was recorded as a potential confounding variable.

\section{Procedure}

Participants first completed the DDNSI, BDI, rRST BIS Anxiety, and PSQI as part of an online questionnaire. They were subsequently invited to participate in the experimental study. Upon presentation to the study (no later than 1 week from completion of questionnaire), participants completed the nightmare recall questionnaire, indicated their highest mathematical qualification and undertook the PVSAT-C. Full written debrief was provided on completion of the study.

\section{Statistical Analysis}

Preliminary univariate statistical tests were conducted on all study dependent variable and covariates. Two analyses of covariance (ANCOVAs) were subsequently performed testing the hypothesis that nightmare group participants would be less tolerant of stress (shorter PVSAT-C termination latency) than controls. Group was entered as the factor. PVSAT-C duration (stress tolerance) was entered as the dependent variable. Our first model restricted the amount of parameters being controlled for to obtain an indication of the effects of frequent nightmares while only controlling for a nightmare prior to the study allowing for trait effects to be detected while controlling for potentially confounding state effects of nightmares. The second model entered additional covariates; depressive symptoms (BDI), global sleep quality (PSQI), trait anxiety (rRST BIS Anxiety), and highest mathematical qualification. 
Table 1

Descriptive Statistics for Control and Frequent Nightmare Groups

\begin{tabular}{|c|c|c|c|c|}
\hline & $\begin{array}{l}\text { Controls }(n=42) \\
\text { Mean }(S D)\end{array}$ & $\begin{array}{c}\text { Frequent nightmare } \\
(n=43) \\
\text { Mean }(S D)\end{array}$ & $t / \chi^{2}$ & $p$ \\
\hline PVSAT-C duration (s) & $229.54(229.36)$ & $157.09(184.33)$ & 1.61 & .112 \\
\hline Depressive symptoms & $9.81(7.48)$ & $17.86(9.75)$ & -4.26 & $<.001$ \\
\hline Global sleep quality & $5.81(2.11)$ & $9.60(3.41)$ & -6.16 & $<.001$ \\
\hline Trait anxiety & $3.17(.67)$ & $3.45(.62)$ & -2.05 & .043 \\
\hline Nightmare recall (on day of study) ${ }^{\mathrm{a}}$ & 3 & 21 & 16.69 & $<.001$ \\
\hline Math qualification ${ }^{b}$ & & & 4.68 & .322 \\
\hline None $(0)$ & 0 & 1 & & \\
\hline GCSE (1) & 22 & 24 & & \\
\hline AS-level (2) & 7 & 3 & & \\
\hline A-level (3) & 13 & 13 & & \\
\hline 1st degree (4) & 0 & 2 & & \\
\hline
\end{tabular}

Note. $\quad$ PVSAT-C $=$ Paced Visual Serial Addition Task-Computerized; GCSE $=$ General Certificate of Secondary Education.

${ }^{\mathrm{a}}$ Binomial and ${ }^{\mathrm{b}}$ multinomial variables are expressed as count data.

\section{Results}

Descriptive statistics for the sample are displayed in Table 1. Means and standard deviations $(S D)$ are provided for interval and ordinal data while frequency counts are provided for categorical data. Preliminary univariate analyses are also reported in Table 1. These indicated no significant difference between the nightmare and control group on mathematical qualification. However, nightmare recall (nightmare on day of study) was significantly greater in the nightmare group. Nightmare group participants also reported greater levels of depressive symptoms than controls, lower sleep quality, and higher trait anxiety.

An ANCOVA first examined the effect of nightmare level on stress tolerance while controlling for confounding state effect of nightmare prior to the study. While the covariate showed a nonsignificant effect, $F(1,82)=2.62, p=.110, \eta_{\text {par }}^{2}=.031$, a significant difference between nightmare groups on stress tolerance was observed, $F(1,82)=4.72, p=.033, \eta_{\text {par }}^{2}=.054$, indicating that participants experiencing frequent nightmares had shorter latencies for forcibly exiting the stress task compared to control participants.

A second ANCOVA examined the effect of nightmare level on stress tolerance while controlling for confounding state effect of nightmare prior to the study, $F(1,78)=2.79, p=.099, \eta_{\text {par }}^{2}=.035$, depressive symptoms, $F(1,78)=.16$, $p=.687, \eta_{\text {par }}^{2}=.002$, global sleep quality, $F(1,78)=.43, p=.513, \eta_{\text {par }}^{2}=.006$, trait anxiety, $F(1,78)=.54, p=.466, \eta_{\text {par }}^{2}=.007$, and mathematics qualification, $F(1$, $78)=.82, p=.367, \eta_{\text {par }}^{2}=.010$. A significant difference between nightmare groups on stress tolerance was observed, $F(1,78)=7.17, p=.009, \eta_{\mathrm{par}}^{2}=.084$, remained upon controlling for covariates.

\section{Discussion}

Our results indicate that individuals experiencing frequent nightmares ended the PVSAT-C task significantly sooner than controls. As such, individuals reporting 
frequent nightmares appear to have significantly lower tolerance to stressor than those individuals reporting infrequent nightmares. These findings support our hypothesis, prior literature (Blagrove et al., 2004; Köthe \& Pietrowsky, 2001; Lancee \& Schrijnemaekers, 2013) and predictions of the AND model (Nielsen \& Levin, 2007) that individuals experiencing frequent nightmares are more susceptible to daily stressors. Thus, the present study has replicated and extended the existing literature by using objective measures of stress, the PVSAT-C (Lejuez et al., 2003). This builds on the existing literature in this domain which largely employs self-report measures of stress. Furthermore, the observed effect remained and increased (from $\eta_{\text {par }}^{2}=.054$ for Model 1 to $\eta_{\text {par }}^{2}=.084$ for Model 2) when controlling for the effects of covariates: depression symptoms, global sleep quality, occurrence of an acute nightmares event directly prior to the study, trait anxiety, and a task specific covariate mathematical ability. This indicates that the trait effects of nightmares are pervasive and influence stress tolerance above and beyond common factors known to increase vulnerability to stressors.

These findings should be treated as tentative, due to the gender imbalance (85.9\% female) and limited age range (18-31) of our student sample. Additionally the limited age range of the sample allows for inferences to be made regarding young adults only. Nightmare frequency may fluctuate with age-indeed studies have reported reductions (Schredl, 2013), increases (Sandman et al., 2013) but also no change (Schredl, 2010) with age. Hence, it is unclear how limiting our sample's age range is for the generalizability of our findings, and so replication with a variety of ages may be useful in informing the targeting of vulnerable groups for interventions.

Though clear differences in stress tolerance can be observed as a function of experiencing frequent nightmares, our results do not establish causality. Namely, if nightmares directly decrease stress tolerance or if this effect is due to participants having elevated baseline stress levels resulting in more frequent nightmares as a by-product. Alternatively, it is possible that the frequent nightmare group consisted of individuals with a generally lower stress tolerance threshold, or lower cognitive function making participation in a cognitively demanding task more stressful. Longitudinal research on the directionality of the effects of nightmares on daily stress tolerance is now required. Additionally, replications with baseline measures of stress and cognitive abilities would help account for these potential confounds.

While these limitations require consideration, our findings do replicate trends of the established literature indicating frequent nightmares to be associated with reduced stress resilience (Blagrove et al., 2004; Blagrove \& Haywood, 2006; Lancee \& Schrijnemaekers, 2013). Such a negative association between stress resilience and frequent nightmares suggests intervention may benefit those frequently afflicted by nightmares. Reduced stress tolerance linked to frequent nightmares has also been hypothesized to underpin the mechanism linking nightmares to suicidality (Bernert \& Joiner, 2007). Thus, we suggest individuals experiencing elevated frequency of nightmares may benefit from nightmare reducing interventions such as imagery rehearsal therapy (IRT; Krakow \& Zadra, 2010). IRT asks clients to recall a nightmare, write down the content, and deliberately alter the nightmare's negative ending or theme into more pleasant scenario which can then be rehearsed to displace the unwanted content (Krakow et al., 2001). IRT is suggested as first line psychological treatment for nightmare disorder (Aurora et al., 2010; Cranston, 
Davis, Rhudy, \& Favorite, 2011). However, should nightmare treatments be unavailable, treatments aimed at increasing stress tolerance may be helpful in mitigating nightmares' associated effect on stress tolerance. Specifically, acceptance and commitment therapy (ACT; Hayes, Strosahl, \& Wilson, 1999) based treatments have shown promise in increasing individuals' ability to tolerate distress related to a number of behaviors (Ruiz, 2010). ACT aims to promote psychological flexibility, the ability to remain in the present moment while accepting the presence of aversive events rather than controlling them to engage in actions recognized as being of value to the individual. Such a treatment would be beneficial since it may, in turn, reduce nightmare frequency due to diminished daily stress levels.

Our findings highlight frequent nightmares being associated with reductions in distress tolerance tested with a behavioral task. Bearing in mind the implications of diminished stress tolerance as suggested by Bernert and Joiner (2007), and the availability of effective treatments, clinicians should consider nightmares as a target for intervention.

\section{References}

American Academy of Sleep Medicine. (2014). International classification of sleep disorders (3rd ed.). Darien, IL: Author.

Aurora, R. N., Zak, R. S., Auerbach, S. H., Casey, K. R., Chowdhuri, S., Karippot, A., . . . the American Academy of Sleep Medicine. (2010). Best practice guide for the treatment of nightmare disorder in adults. Journal of Clinical Sleep Medicine: JCSM: Official Publication of the American Academy of Sleep Medicine, 6, 389-401.

Beck, A. T., Steer, R. A., \& Brown, G. K. (1996). Manual for the Beck Depression Inventory-II. San Antonia, TX: Psychological Corporation.

Bernert, R. A., \& Joiner, T. E. (2007). Sleep disturbances and suicide risk: A review of the literature. Neuropsychiatric Disease and Treatment, 3, 735-743.

Blagrove, M., Farmer, L., \& Williams, E. (2004). The relationship of nightmare frequency and nightmare distress to well-being. Journal of Sleep Research, 13, 129-136.

Blagrove, M., \& Haywood, S. (2006). Evaluating the awakening criterion in the definition of nightmares: How certain are people in judging whether a nightmare woke them up? Journal of Sleep Research, 15, 117-124. http://dx.doi.org/10.1111/j.1365-2869.2006.00507.x

Buysse, D. J., Reynolds, C. F., III, Monk, T. H., Berman, S. R., \& Kupfer, D. J. (1989). The Pittsburgh Sleep Quality Index: A new instrument for psychiatric practice and research. Psychiatry Research, 28, 193-213. http://dx.doi.org/10.1016/0165-1781(89)90047-4

Cranston, C. C., Davis, J. L., Rhudy, J. L., \& Favorite, T. K. (2011). Replication and expansion of "best practice guide for the treatment of nightmare disorder in adults." Journal of Clinical Sleep Medicine, 7, 549-553.

Daughters, S. B., Lejuez, C. W., Strong, D. R., Brown, R. A., Breen, R. B., \& Lesieur, H. R. (2005). The relationship among negative affect, distress tolerance, and length of gambling abstinence attempt. Journal of Gambling Studies, 21, 363-378. http://dx.doi.org/10.1007/s10899-005-5554-6

Hayes, S. C., Strosahl, K., \& Wilson, K. G. (1999). Acceptance and committement therapy: An experiential approach to behavior change. New York, NY: Guilford Press.

Heym, N., Skatova, A., Ferguson, E., \& Lawrence, C. (2015). The development and psychometric evaluation of the RST subsystems scales for the measurement of approach and avoidance motivation. Manuscript submitted for publication.

Hochard, K. D., Heym, N., \& Townsend, E. (2015). The unidirectional relationship of nightmares on self-harmful thoughts and behaviors. Dreaming, 25, 44-58. http://dx.doi.org/10.1037/a0038617

Köthe, M., \& Pietrowsky, R. (2001). Behavioral effects of nightmares and their correlations to personality patterns. Dreaming, 11, 43-52. http://dx.doi.org/10.1023/A:1009468517557

Krakow, B. (2006). Nightmare complaints in treatment-seeking patients in clinical sleep medicine settings: Diagnostic and treatment implications. Sleep: Journal of Sleep and Sleep Disorders Research, 29, 1313-1319.

Krakow, B., Hollifield, M., Johnston, L., Koss, M., Schrader, R., Warner, T. D., . . Prince, H. (2001). Imagery rehearsal therapy for chronic nightmares in sexual assault survivors with posttraumatic 
stress disorder: A randomized controlled trial. JAMA: Journal of the American Medical Association, 286, 537-545. http://dx.doi.org/10.1001/jama.286.5.537

Krakow, B., \& Zadra, A. (2010). Imagery rehearsal therapy: Principles and practice. Sleep Medicine Clinics, 5, 289-298. http://dx.doi.org/10.1016/j.jsmc.2010.01.004

Lancee, J., \& Schrijnemaekers, N. C. (2013). The association between nightmares and daily distress. Sleep and Biological Rhythms, 11, 14-19. http://dx.doi.org/10.1111/j.1479-8425.2012.00586.x

Lejuez, C. W., Kahler, C. W., \& Brown, R. A. (2003). A modified computer version of the Paced Auditory Serial Addition Task (PASAT) as a laboratory-based stressor. The Behavior Therapist, 26, 290-293.

Leproult, R., Copinschi, G., Buxton, O., \& Van Cauter, E. (1997). Sleep loss results in an elevation of cortisol levels the next evening. Sleep: Journal of Sleep and Sleep Disorders Research, 20, 865-870.

Nielsen, T., \& Levin, R. (2007). Nightmares: A new neurocognitive model. Sleep Medicine Reviews, 11, 295-310. http://dx.doi.org/10.1016/j.smrv.2007.03.004

Open Science Collaboration. (2015). Estimating the reproducibility of psychological science. Science, 349, aac4716. http://dx.doi.org/10.1126/science.aac4716

Ruiz, F. J. (2010). A Review of acceptance and commitment therapy (ACT) empirical evidence: Correlational, experimental psychopathology, component and outcome studies. International Journal of Psychology \& Psychological Therapy, 10, 125-162.

Sandman, N., Valli, K., Kronholm, E., Ollila, H. M., Revonsuo, A., Laatikainen, T., \& Paunio, T. (2013). Nightmares: Prevalence among the Finnish general adult population and war Veterans during 1972-2007. Sleep: Journal of Sleep and Sleep Disorders Research, 36, 1041-1050.

Schredl, M. (2010). Nightmares: An under-diagnosed and undertreated condition? Sleep: Journal of Sleep and Sleep Disorders Research, 33, 733-734.

Schredl, M. (2013). Nightmare frequency in a representative German sample. International Journal of Dream Research, 6, 119-122.

Tombaugh, T. N. (2006). A comprehensive review of the Paced Auditory Serial Addition Test (PASAT). Archives of Clinical Neuropsychology, 21, 53-76. http://dx.doi.org/10.1016/j.acn.2005.07.006 